\title{
Renormalization of correlations in a quasiperiodically forced two-level system for a general class of modulation function
}

\author{
L N C Adamson* and A H Osbaldestin ${ }^{\dagger}$ \\ Department of Mathematics \\ University of Portsmouth \\ Portsmouth \\ PO1 3HF, UK
}

November 16, 2014

\begin{abstract}
We provide a renormalization analysis of correlations in a quasi-periodically forced two-level system in a time dependent field with periodic kicks whose amplitude is given by a general class of discontinuous modulation function. For certain intensities of modulation, we give a complete understanding of the autocorrelation function. Furthermore, once the locations of the discontinuities of the modulation function are known, aperiodic orbits lead to correlations on renormalization strange sets which are determined by two specified features of the modulation function of which there are only a finite number of variations.
\end{abstract}

PACS: 05.45.-a; 64.60.ae

\section{Introduction}

The motivation of this paper is to further understand correlations in a two-level quantum system which is subject to kicks with quasiperiodically modulated amplitude. The system has been previously studied in [6], the results of which were then expanded upon in [12] and [15]. The system was originally examined in [10], where its response to quasiperiodic perturbations is considered. It is shown that the Fourier transform of the evolution operator is not quasiperiodic, and represents an intermediate state between quasiperiodic and chaotic motion.

In [6] the system is analysed subject to kicks with quasiperiodically modulated amplitude, where the driving frequency is the inverse of the golden mean $(\sqrt{5}-1) / 2$. If the modulation function is chosen to be continuous, quasiperiodic behaviour of the autocorrelation function $(\mathrm{ACF})$ is observed, whereas if the modulation function is piece-wise constant then the ACF neither decays to zero or returns to 1 . In this case the variable amplitude is taken to be $A_{n}=\kappa \Phi\left(\phi_{n}\right)$, where $\Phi$ is a piece-wise constant function, $\kappa$ denotes the intensity or "level" of modulation and $\phi_{n}$ is a simple quasiperiodic rotation on a circle. In [6] it is numerically determined that for $\kappa=\pi / 2$ and $\Phi$ as given in (4.1) (with $\alpha=1 / 2$ ), the ACF is self-similar near each main peak as shown in Figure 1. It is numerically observed that the peaks are of approximate magnitude 0.55279. In [12] the authors provided a complete analysis of all piece-wise constant periodic orbits of the additive functional recurrence which appears in the renormalization analysis of the system. For the modulation function (4.1) and $\kappa=\pi / 2$, it is analytically shown in [12] that the

\footnotetext{
*luke.adamson@port.ac.uk

† andrew.osbaldestin@port.ac.uk
} 


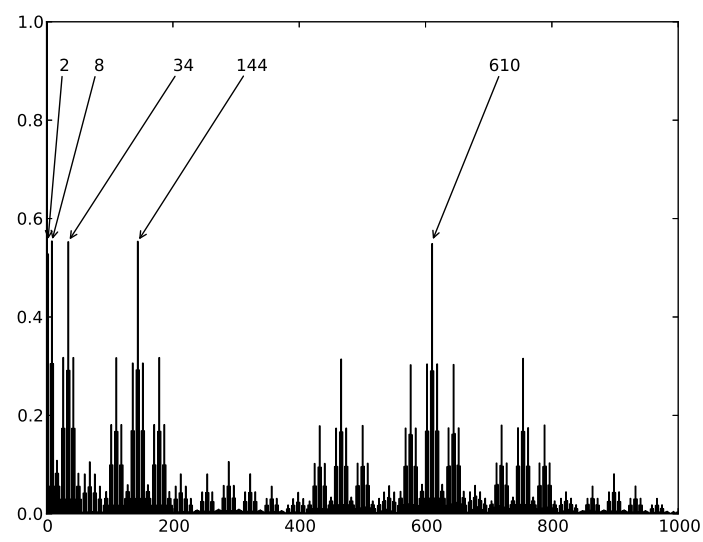

Figure 1: Numerical evaluation of the auto correlation $C_{P_{y}}$ for modulation function (4.1) with modulation level $\kappa=\pi / 2$.

peaks occur at times $F_{3 k}$ (where $F_{n}$ is the Fibonacci sequence) with asymptotic height $1-1 / \sqrt{5}=0.5527 \ldots$ The authors extended the results in [15] to a class of quadratic irrational frequencies.

These properties of the ACF are indicative of a class of systems exhibiting singular continuous spectra. Indeed, similar phenomenon has been observed in studies of symmetric barrier billiards ([21],[3],[17],[2]), localized eigenstates of the generalised Harper equation $([8],[16],[14],[13])$ and the transition to, and correlations of, strange non-chaotic attractors (SNAs) ([9],[5],[11],[18],[1]).

In the study of symmetric barrier billiards, the Fourier transform of the observable is shown in [21] to exhibit singular continuous spectra and, for the golden mean trajectory, the autocorrelation function is shown to have peaks of magnitude $\simeq 0.55$ for the half barrier. In [3] a renormalization analysis for barrier billiards is provided, giving a thorough treatment to piece-wise constant periodic orbits of the functional recurrence

$$
\tilde{Q}_{n}(x)=\tilde{Q}_{n-1}(-\omega x) \tilde{Q}_{n-2}\left(\omega^{2} x+\omega\right),
$$

which leads to self-similar behaviour of the ACF, and it is shown that the peaks occur at every third Fibonacci number and are of magnitude $1-1 / \sqrt{5}=0.5527 \ldots$ with alternating sign in the case of the half barrier for the golden mean trajectory. As a matter of fact the renormalization operator (1.1) is the multiplicative version of (3.7) which will be the main focus of this paper. It is thus no surprise that the structure of the ACF in the two systems is similar.

This work is extended by us in [17] to cases when the modulation function leads to aperiodic behaviour of the renomalization operator, and it is found that the correlations lie on an invariant renormalization strange set embedded in three dimensional space, and are also chaotic at Fibonacci times. In [2] we extended further to a class of quadratic irrational trajectories (as was done in [15] for the two-level system under study here), analytically providing the locations and magnitudes of the peaks in the ACF for the half barrier and numerically producing the corresponding renormalization strange sets in the aperiodic case.

A renormalization analysis of the localized eigenstates of Harper equation was provided in [8] and gave rise once more to the recurrence (1.1) in the case of inverse golden mean flux. The authors discovered the existence of a strong coupling fixed point of this functional recurrence, which was later constructed fully in [16] and then extended to a class of quadratic irrational in [4]. The authors also studied the generalised Harper equation, showing that the fluctuations of the localized eigenstates are characterized by orbits of the renormalization operator on a renormalization strange set dubbed the "Ketoja-Satija orchid". In [14] the orchid was rigorously constructed via a conjugacy with a Bernoulli shift of finite type, and these results were once again extended to a class of quadratic irrational flux [13]. 
Finally, the multiplicative recurrence (1.1) is once again seen in the study of both the correlations and bifurcations of SNAs. In [9] the so-called "blow-out birth of an SNA" is studied for the inverse golden mean frequency, and it is shown that at and near the critical boundary the attractor is self-similar following scaling laws determined by the renormalization equations. We have extended this study in [1] to the more general non-smooth pitchfork bifurcation. In [11] the renormalization of correlations of the original SNA first discovered in [7] are studied. It is shown that periodic behaviour of the multiplicative recurrence once again drives the self-similar behaviour of the ACF, which has the same properties as the ACF derived for barrier billiards. In [1] a direct link between the correlations of these two systems is made.

We begin by describing the two-level system under study and deriving the relevant renormalization equations in Section 2. In Section 3 we look at the renormalization of the ACF and give the result which makes most of the work in this paper possible. In Section 4 we examine modulation functions leading to periodic behaviour of the ACF, and eliminate the issue of unbounded orbits of the renormalization operator. In Section 5 we examine modulation functions leading to aperiodic behaviour of the renormalization operator and chaotic correlations lying on renormalization strange sets. Section 6 focuses on varying the level of modulation to examine its effect on the resulting renormalization strange sets.

\section{Derivation of the renormalization equations}

Following [5] and [12], for a two-level system in a time-dependent magnetic field $M(t)$, the Hamiltonian takes the form $H(t)=M(t) \cdot \sigma$ where $\sigma=\left(\sigma_{x}, \sigma_{y}, \sigma_{z}\right)$, the components of this vector being given by the Pauli matrices

$$
\sigma_{x}=\left(\begin{array}{cc}
0 & 1 \\
1 & 0
\end{array}\right), \quad \sigma_{y}=\left(\begin{array}{cc}
0 & -i \\
i & 0
\end{array}\right), \quad \sigma_{z}=\left(\begin{array}{cc}
1 & 0 \\
0 & -1
\end{array}\right) .
$$

In the case when $M(t)=(S(t) / 2,0, k / 2)$ we have $H(t)=(S(t) / 2) \sigma_{x}+(k / 2) \sigma_{z}$. Setting Planck's constant $\hbar=1$ we can write the Schrödinger equation for a spinor $\psi=\left(\psi_{1}, \psi_{2}\right)$ in terms of the observable Bloch variables. These are the components of the polarization vector $P_{x, y, z}=\psi^{*} \sigma_{x, y, z} \psi$, from which we obtain the following differential equations

$$
\begin{aligned}
& \dot{P}_{x}=-k P_{y}, \\
& \dot{P}_{y}=k P_{x}-S(t) P_{z}, \\
& \dot{P}_{z}=S(t) P_{y},
\end{aligned}
$$

which are normalized so that $P_{x}^{2}+P_{y}^{2}+P_{z}^{2}=1$. We restrict our attention to the case when $S(t)$ consists of period- $T$ $\delta$-function kicks i.e

$$
S(t)=\sum_{n=-\infty}^{\infty} A_{n} \delta(t-n T),
$$

where the amplitude $A_{n}$ is variable. In between the kicks we therefore have a rotation in the $\left(P_{x}, P_{y}\right)$ plane, and at the kicks a rotation in the $\left(P_{y}, P_{z}\right)$ plane resulting in the following "kick-to-kick" linear mapping

$$
\left(\begin{array}{l}
P_{x, n+1} \\
P_{y, n+1} \\
P_{z, n+1}
\end{array}\right)=\left(\begin{array}{ccc}
\cos k T & -\sin k T \cos A_{n} & \sin k T \sin A_{n} \\
\sin k T & \cos k T \cos A_{n} & -\cos k T \sin A_{n} \\
0 & \sin A_{n} & \cos A_{n}
\end{array}\right)\left(\begin{array}{l}
P_{x, n} \\
P_{y, n} \\
P_{z, n}
\end{array}\right) .
$$

The notation $\left(P_{x, n}, P_{y, n}, P_{z, n}\right)$ denotes the polarization vector at each such time step $n$. As in [5] we now take $A_{n}=\kappa \Phi\left(\phi_{n}\right)$, where $\Phi$ is the modulation function, $\phi_{n}$ is a quasiperiodic rotation i.e. $\phi_{n+1}=\phi_{n}+\omega$ with $\omega \notin \mathbb{Q}$, and $\kappa$ is a constant determining the amplitude of the modulation. Throughout this paper we will focus on piecewise constant modulation functions, where singular-continuous spectra is observed as shown in [10].

We now set the time $T$ between kicks to be commensurate with the fundamental frequency $k$ so that $k T=2 \pi m$, $m \in \mathbb{Z}$. This effectively decouples $P_{x, n}$ so that the remaining dynamics is just a rotation in the $\left(P_{y}, P_{z}\right)$ plane. Note that it is numerically seen in [6] that this simplification may not be necessary to observe singular continuous 
spectra. As a result of the normalization constraint we may without loss of generality take $P_{x, n}=0$. Writing $P_{y, n}=\cos \theta_{n}$ and $P_{z, n}=\sin \theta_{n}$ results in the following skew-product system

$$
\begin{aligned}
\phi_{n+1} & =\phi_{n}+\omega \quad(\bmod 1), \\
\theta_{n+1} & =\theta_{n}+\kappa \Phi\left(\phi_{n}\right) .
\end{aligned}
$$

This system is of the form also seen in the study of strange non-chaotic attractors (SNAs) [5] and symmetric barrier billiards [21]. We can "solve" the system (2.7)-(2.8) in straightforward fashion giving

$$
\begin{gathered}
\phi_{n}=\phi_{0}+n \omega \quad(\bmod 1), \\
\theta_{n}=\theta_{0}+\kappa \sum_{l=0}^{n-1} \Phi\left(\phi_{0}+l \omega\right) .
\end{gathered}
$$

This solution does not however give any insight as to the behaviour of $\theta$ and so we analyse the autocorrelation function.

\section{Renormalization of the autocorrelation function}

The normalized autocorrelation function for the observable $P_{y}$ is given by

$$
C_{P_{y}}(t)=\frac{\left\langle P_{y, n} P_{y, n+t}\right\rangle}{\left\langle P_{y, n}^{2}\right\rangle}
$$

where $\langle f(n)\rangle=\lim _{N \rightarrow \infty} \frac{1}{N} \sum_{n=1}^{N} f(n)$. Making use of the trigonometric identity $2 \cos A \cos B=\cos (A+B)+\cos (A-B)$ we see that

$$
\left\langle P_{y, n} P_{y, n+t}\right\rangle=\left\langle\cos \theta_{n} \cos \theta_{n+t}\right\rangle=\frac{1}{2}\left\langle\cos \left(\theta_{n+t}-\theta_{n}\right)\right\rangle=\frac{1}{2}\left\langle\cos \kappa S_{t}\left(\phi_{n}\right)\right\rangle,
$$

where we average over the initial phase to remove $\left\langle\cos \left(\theta_{n+t}+\theta_{n}\right)\right\rangle$ and define

$$
S_{t}(\phi)=\sum_{i=0}^{t-1} \Phi(\phi+i \omega), \quad S_{0}(\phi)=0 .
$$

Using the ergodicity in $\phi$ we can now write

$$
C_{P_{y}}(t)=\left\langle\cos \kappa S_{t}\left(\phi_{n}\right)\right\rangle=\int_{0}^{1} \cos \kappa S_{t}(\phi) \mathrm{d} \phi .
$$

From now on we set $\omega$ to be the inverse of the golden mean, $\omega=(\sqrt{5}-1) / 2$ and consider the autocorrelation function $(\mathrm{ACF})$ at Fibonacci times. It is straightforward to show that

$$
S_{F_{n}}(\phi)=S_{F_{n-1}}(\phi)+S_{F_{n-2}}\left(\phi+F_{n-1} \omega\right),
$$

where the Fibonacci numbers $F_{n}$ satisfy $F_{n+1}=F_{n}+F_{n-1}(n \geq 2)$ with $F_{0}=0, F_{1}=1$. Defining the rescaled functions

$$
Z_{n}(x)=S_{F_{n}}\left(x(-\omega)^{n}\right),
$$

and making use of the identity $F_{n-1} \omega=F_{n-2}-(-\omega)^{n-1}$ gives the functional recurrence

$$
Z_{n}(x)=Z_{n-1}(-\omega x)+Z_{n-2}\left(\omega^{2} x+\omega\right), \quad Z_{0}(x)=0, Z_{1}(x)=\Phi(-\omega x) .
$$

Substituting this change of variables into (3.4) we obtain that the ACF at Fibonacci times is given by

$$
C_{P_{y}}\left(F_{n}\right)=\frac{1}{(-\omega)^{-n}} \int_{0}^{(-\omega)^{-n}} Q_{n}(x) \mathrm{d} x
$$


where $Q_{n}(x)=\cos \kappa Z_{n}(x)$.

We note in (3.8) that as $n$ increases the range over which we must integrate is unbounded, and so for numerical work we need to resort to approximations over a finite interval. Numerically we observe that taking an interval $[-X, X]$ and integrating $\cos \kappa Z_{n}(x)$ over it leads to convergence for $X$ sufficiently large and so we may fix our attention to $Z_{n}$ over such an interval. Typically we find $X=150$ to provide a good balance between accuracy and computation time. In order to calculate this average however, we need a knowledge of the value of $Z_{n}$ at $-X$ (or any other point, but the value at $-X$ is convenient as we integrate from left to right) in addition to both the locations and the sizes of the discontinuities. It is straightforward to see that $Z_{n}(-X)$ satisfies

$$
Z_{n}(-X)=Z_{n-1}(-X)+Z_{n-2}(-X)+\sum_{x \in[-X, \omega X]} D_{n-1}(x)+\sum_{x \in\left[-X, \omega-\omega^{2} X\right]} D_{n-2}(x),
$$

where we define the discontinuity function $D_{n}(x)=Z_{n}(x+)-Z_{n}(x-)$. When evaluated at a discontinuity, this formula is the definition of the size of the discontinuity.

\section{Modulation functions which give rise to periodic behaviour}

Focusing now on periodic behaviour of the functional recurrence, it follows from the work in [12] and [3] that any modulation function of the form

$$
\Phi(\phi)= \begin{cases}+1, & 0 \leq\{\phi\}<\alpha \\ -1, & \alpha \leq\{\phi\}<1,\end{cases}
$$

will give rise to periodic behaviour of (3.7) if, and only if, $\alpha \in \mathbb{Q}(\omega)(\{\phi\}$ denotes the fractional part of $\phi)$. As $\kappa$ is extrinsic to the behaviour of (3.7) we deduce from (3.8) that any such choice will lead to self-similarity of the ACF. We note that throughout most of this work we will assume that $\Phi$ (and hence the initial condition $Z_{1}$ ) has two discontinuities in order to simplify the analysis and provide some intuition towards the nature of the renormalization strange sets we discover. This means both discontinuities must be of equal magnitude, because otherwise an extra discontinuity at zero is introduced. This assumption allows us to link this work to our previous studies on symmetric barrier billiards.

We now recall the following two propositions from [12], which tell us that for modulation functions leading to periodic behaviour, an understanding of the initial conditions in a "fundamental interval" is sufficient to build the structure of the orbit on $\mathbb{R}$.

Proposition 1. Let $Z_{n}$ be a piecewise-constant periodic orbit of (3.7) with period $p$ on $\mathbb{R}$ with $Z_{n}(1+)=Z_{n}(1)$. Then $Z_{n}$ is periodic with period $p$ on the fundamental interval $I=[-\omega, 1]$. Conversely, suppose that $Z_{n}$ is periodic with period $p$ on $I$. Then there exists a unique extension $\tilde{Z}_{n}$ of $Z_{n}$ such that $\tilde{Z}_{n}$ is periodic with period $p$ on $\mathbb{R}$.

Proposition 2. Let $Z_{0}, Z_{1}$ be piecewise-constant initial conditions for (3.7) on $\mathbb{R}$ with $Z_{0}(1+)=Z_{0}(1), Z_{1}(1+)=$ $Z_{1}(1)$. Suppose $Z_{n}$ is periodic with period $p$ on the fundamental interval $I$. Then the sequence $Z_{n}$ converges to the unique periodic extension $\tilde{Z}_{n}$ given by Proposition 1, i.e., for all integers $r \geq 0$ we have $Z_{r+n p}(x) \rightarrow \tilde{Z}_{r}(x)$ as $n \rightarrow \infty$.

Hence an initial condition on the fundamental interval which results in periodic behaviour uniquely determines an asymptotic (right continuous at 1 ) global periodic orbit on $\mathbb{R}$.

\subsection{Application to previous studies}

In [6] the authors analysed this system for the modulation function given by (4.1) when $\alpha=1 / 2$, where iteration of (3.7) gives rise to a periodic six orbit as shown in Figure 2. Note that $Z_{1}(-X)=+1$ as we only require a knowledge of $Z_{1}(x)=\Phi(-\omega x)$ on the fundamental interval. Indeed, the discontinuities of $Z_{1}$ on the fundamental interval are 
at 0 and $-\omega^{-1}(\alpha-1)$, and we discard the rest of the discontinuities giving $Z_{1}(-X)=+1$. This argument will be used throughout this paper and from now on the value of $Z_{1}(-X)$ will be stated and left to the reader to verify.
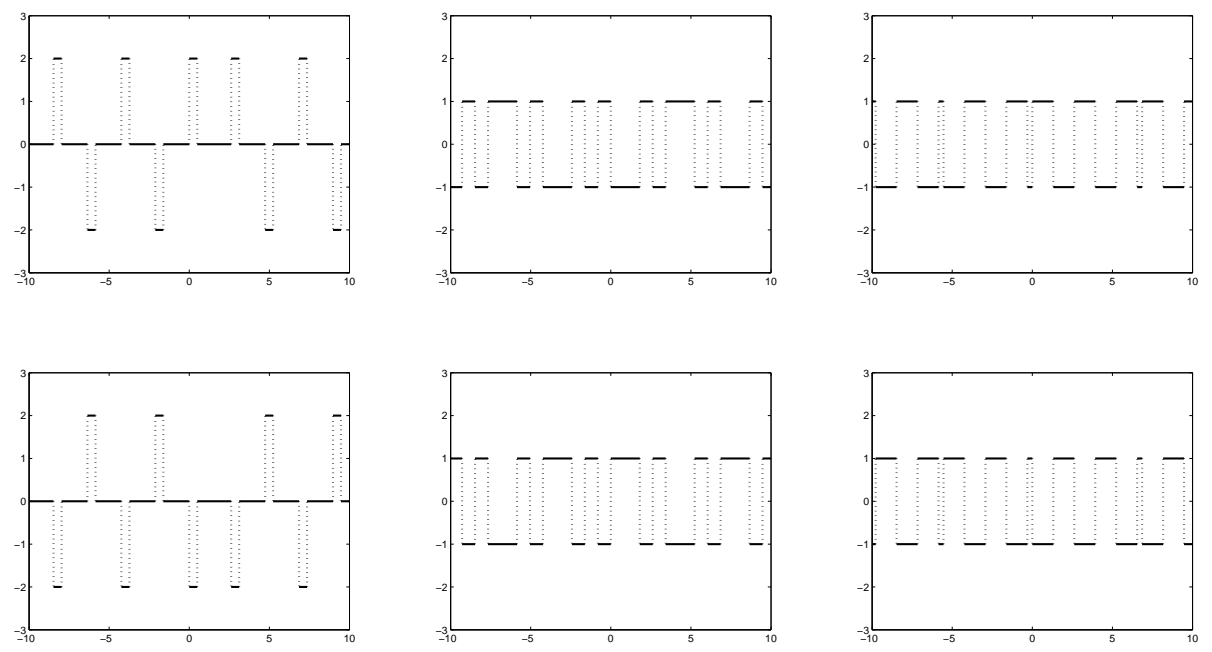

Figure 2: Period 6 orbit of the recurrence (3.7) for choice of modulation function (4.1). First row gives $Z_{0}, Z_{1}, Z_{2}$ and second row gives $Z_{3}, Z_{4}, Z_{5}$

We note that this orbit is clearly bounded, and more generally in [12] sufficient criteria for $Z_{n}$ to be globally bounded were derived. For the choice of $\kappa=\pi / 2$ it was numerically observed that the peaks in the resulting autocorrelation function are of approximate magnitude 0.55279 [6]. In [12] the value is shown to be $1-1 / \sqrt{5}=$ $0.5527864 \ldots$ through careful study of the locations and interlacing of the discontinuities. In particular it is shown that $C_{P_{y}}\left(F_{3 k}\right)=1-1 / \sqrt{5}$ and that $C_{P_{y}}\left(F_{n}\right)$ is zero for $n \equiv 1,2(\bmod 3)$, as can clearly be seen in Figure 1 where the theoretical peaks are indicated by the arrows.

This last fact can be verified easily using (3.9). Note that as the modulation function has only two discontinuities of magnitude two, all future iterates of (3.7) will have discontinuities of magnitude two only. Therefore the function $Z_{n}(x)$ will be only odd or only even for all $x$, and we can deduce this parity through calculation of $Z_{n}(-X)$. As $Z_{n}$ has discontinuities of magnitude two only, the sums on the right hand side of (3.9) will be even, and so the parity of $Z_{n}(-X)$ is determined solely by the first two terms on the right hand side which is the aforementioned Fibonacci recurrence. Noting that $Z_{0}(-X)$ is even and $Z_{1}(-X)$ is odd we thus have that $Z_{2}(-X)$ is odd, and $Z_{3}(-X)$ is even. Continuing in this way we deduce that $Z_{n}(-X)$ (and hence $\left.Z_{n}(x)\right)$ is even only when $n \equiv 0(\bmod 3)$.

We now examine the function $\cos \left(\frac{\pi}{2} Z_{n}(x)\right)$ (recalling that $\left.\kappa=\pi / 2\right)$. As $Z_{n}(x)$ is odd for all $x$ when $n \equiv 1,2$ (mod 3), this function is identically zero and hence from (3.8) the ACF $C_{P_{y}}\left(F_{n}\right)$ is also zero.

For the case when $n \equiv 0(\bmod 3)$ we have to be more subtle. Our initial condition is a function containing two discontinuities, and upon iteration of (3.7) the number of discontinuities grows filling intervals of increasing length. The sum of the sizes of the discontinuities on $Z_{1}$ is zero and so for all future iterates the sum of the sizes of discontinuities is also zero. However, for any $n$ we can always find an $X$ big enough so that all discontinuities of $Z_{n-1}$ and $Z_{n-2}$ are contained in $[-X, \omega X]$. For an $X$ satisfying this condition we can thus write

$$
Z_{n}(-X)=Z_{n-1}(-X)+Z_{n-2}(-X) .
$$

We note that $\cos (\kappa y)$ is periodic with period $2 \pi / \kappa$, or for the case under study the function is periodic with period 4. Thus given a knowledge of the value of $Z_{n}(-X)$ (which is actually equal to $Z_{n}(X)$ due to the prior assumptions on $X$ ) modulo 4 we can get a full understanding of the dynamics. This also eliminates the problem of the functions becoming unbounded in other cases we will encounter. For the case under study we can see that the value of 


\begin{tabular}{|c|c|c|}
\hline$n$ & $Z_{n}(-X)(\bmod 4)$ & $Q_{n}(-X)$ \\
\hline 1 & 1 & 0 \\
2 & 1 & 0 \\
3 & 2 & -1 \\
4 & 3 & 0 \\
5 & 1 & 0 \\
6 & 0 & +1 \\
7 & 1 & 0 \\
8 & 1 & 0 \\
\hline
\end{tabular}

Table 1: Table giving the values of $Z_{n}(-X)$ modulo 4 and $Q_{n}(-X)$ for modulation function (4.1) with $\alpha=1 / 2$.

\begin{tabular}{|c|c|c|c|}
\hline$n$ & $Z_{n}(-X)(\bmod 4)$ & $Q_{n}(-X)$ & $\tilde{Q}_{n}(-X)$ \\
\hline 1 & 0 & +1 & -1 \\
2 & 0 & +1 & -1 \\
3 & 0 & +1 & +1 \\
4 & 0 & +1 & -1 \\
5 & 0 & +1 & -1 \\
6 & 0 & +1 & +1 \\
\hline
\end{tabular}

Table 2: Table giving the values of $Z_{n}(-X)$ modulo 4 and $Q_{n}(-X)$ for modulation function (4.3).

$Z_{n}(-X)$ modulo 4 manifests repetition with period 6 in Table 1 , which we would of course expect. We also list the values of $Q_{n}(-X)$.

Focusing on the cases when $n \equiv 0(\bmod 3)$ we have that $Z_{n}(-X) \equiv 2(\bmod 4)$ when $n \equiv 3(\bmod 6)$ and $Z_{n}(-X) \equiv$ $0(\bmod 4)$ when $n \equiv 0(\bmod 6)$. At first sight this may not seem overly useful as calculation of the integrals is still required using the methods in [12], but, as we will see in the next subsection, we can use this fact to provide a link between this system and another similar system recently studied.

\subsection{Varying the location of the discontinuities}

It should be clear by now that we have a thorough qualitative understanding of modulation functions leading to periodic behaviour. In short, if we have a modulation function with two discontinuities, both of which lie in $\mathbb{Q}(\omega)$, the resulting behaviour of $Z_{n}$ will be periodic, which leads to self-similarity of the ACF. For reasons which are explained below, we now work with the modulation function given by

$$
\Phi(\phi)= \begin{cases}+2, & \{\phi\} \in[0, \alpha / 2] \cup[1-\alpha / 2,1], \\ 0, & \text { Otherwise. }\end{cases}
$$

As before, $\{\phi\}$ denotes the fractional part of $\phi$. The motivation for this choice is that it makes $Z_{1}(-X)$ even, and so from (4.2) it makes $Z_{n}(-X)$ even for all $n$, which means the ACF will never be trivially zero as in the previous case. This modulation function is related to the one studied in [17] where the focus is symmetric barrier billiards. Indeed, the locations of the discontinuities are identical and the function in that study is merely $\tilde{\Phi}(\phi)=\Phi(\phi)-1$. In [17] the modulation function corresponded to a barrier of height $\alpha$ placed centrally in a rectangular chamber. It was shown that an arbitrary barrier can be decomposed into barriers of this type. Furthermore, the renormalization analysis for that system simply gave rise to the multiplicative version of (3.7). Hence upon iteration of (3.7) the locations of the discontinuities will be identical to those generated in [17]. Keeping $\kappa=\pi / 2$ and $\alpha=1 / 2$, we now produce the analogue of Table 1 for the modulation function (4.3) in Table 2.

In this case the value of $Z_{n}(-X)$ is fixed (note that $Z_{1}(-X)=0$ as we are only interested in discontinuities of $Z_{1}(x)=\Phi(-\omega x)$ in the fundamental interval) and hence so is $Q_{n}(-X)$. Now $\tilde{Q}_{n}(x)$ is the result of iteration of the 
multiplicative version of (3.7) i.e.

$$
\tilde{Q}_{n}(x)=\tilde{Q}_{n-1}(-\omega x) \tilde{Q}_{n-2}\left(\omega^{2} x+\omega\right),
$$

with initial conditions $\tilde{Q}_{0}=1$ and $\tilde{Q}_{1}=\tilde{\Phi}(-\omega x)$ which is the required renormalization for barrier billiards in [17], and the values of $\tilde{Q}_{n}(-X)$ are obtained under iteration of the multiplicative version of (3.9) with initial conditions $\tilde{Q}_{0}(-X)=+1$ and $\tilde{Q}_{1}(-X)=-1$. In [17], because the recurrence is multiplicative and the initial condition takes the values \pm 1 , all future iterates are piecewise constant functions taking the values \pm 1 . The calculation of the autocorrelation function simply relies on changing the integrand to $\tilde{Q}_{n}$ in (3.8).

However, $Q_{n}(x)$ is very similar to $\tilde{Q}_{n}$, in fact they are identical except for a choice of sign. This is because, starting at $Z_{n}(-X)$, every time we hit a discontinuity of $Z_{n}(x)$ we either add or subtract two from the value beforehand. In $Q_{n}(x)$, because $\cos (x \pm \pi)=-\cos (x)$, this simply has the effect of multiplying by -1 every time we hit a discontinuity (recall that $\kappa=\pi / 2$ ). Hence $Q_{n}(x)$ is a piecewise constant function taking the values \pm 1 , with its discontinuities in exactly the same locations as $\tilde{Q}_{n}$. Hence the autocorrelation up to a choice of sign will be identical. We see from Table 2 that the signs of $Q_{n}$ and $\tilde{Q}_{n}$ are different when $n \neq 0(\bmod 3)$. From the results of [3] we see immediately that in these cases $C_{P_{y}}\left(F_{n}\right)=0$ and so the difference in sign is irrelevant. However when $n \equiv 0$ $(\bmod 3)$ the signs agree and so from [3] $C_{P_{y}}\left(F_{3 k}\right) \rightarrow(-1)^{k}(1-1 / \sqrt{5})$.

\section{Modulation functions leading to aperiodic behaviour of the autocor- relation function}

If in the initial modulation function, we have a discontinuity which lies outside the field $\mathbb{Q}(\omega)$ we will witness aperiodic behaviour as the discontinuity locations on the fundamental interval will no longer be periodic [11]. Going back to the example studied in Subsection 4.2, we take $\alpha \notin \mathbb{Q}(\omega)$ and iterate forward using (3.7). There are two different cases for the initial conditions: if $\alpha / 2<\omega^{2}$ then Table 2 is still valid, as is the argument as to the similarity of $Q_{n}$ and $\tilde{Q}_{n}$. Thus the only difference in the autocorrelation will be the sign for $n \neq 0(\bmod 3)$. If $\alpha / 2>\omega^{2}$ then $Z_{1}(-X)=+2$ and the same conclusion is reached noting that in this case $\tilde{Q}_{1}(-X)=+1$, and so the signs in Table 2 for $Q_{n}$ and $\tilde{Q}_{n}$ are simply switched. In [17] for the barrier billiard system we showed that reconstruction of the dynamics in three dimensions by plotting the resulting time series $\left(C_{P_{y}}\left(F_{n}\right)\right)$ using the work of [20] leads to a renormalization strange set. The two dimensional projections are shown in Figure 3. This set embedded in threedimensional space is the same for the two level system and is shown in Figure 4. Using the method of Rosenstein [19] (chosen for its robustness), we numerically calculated a positive largest Lyapunov exponent (LLE) of 0.4136 $( \pm 0.005)$, confirming that the correlations are chaotic at Fibonacci times in [17]. This value and (cautious) error estimate was achieved by finding the mean of the LLEs generated from 25 independent time series each of length 10000. Note that the separation of nearby points may not occur at an exponential rate in real time. The positive Lyapunov exponent is a by-product of the chaotic nature of the renormalization operator (3.7) being reflected in the asymptotic averages of the functions $Q_{n}(x)=\cos \kappa Z_{n}(x)$. The correlations densely explore the renormalization strange set which appears to be a smooth surface with cusps. (They do not fill the space inside.) The fact the surface is connected to only four corners of the unit cube follows from the fact that the autocorrelation function can only be \pm 1 if $Z_{0,1}(x)=0$ or 2 or, equivalently, $\tilde{Q}_{0,1}(x)= \pm 1$ where $\tilde{Q}$ follow recurrence (4.4). This leads to four possible sign triples, three of which lie on the periodic orbit $(+1,-1,-1) \rightarrow(-1,-1,+1) \rightarrow(-1,+1,-1)$ and the other at $(+1,+1,+1)$ is fixed. 


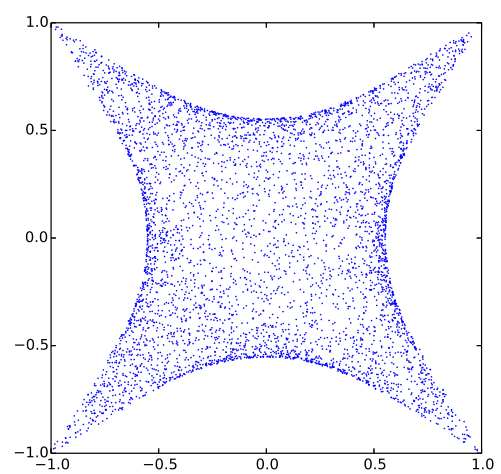

(a) $C_{P_{y}}\left(F_{n+1}\right)$ vs. $C_{P_{y}}\left(F_{n}\right)$

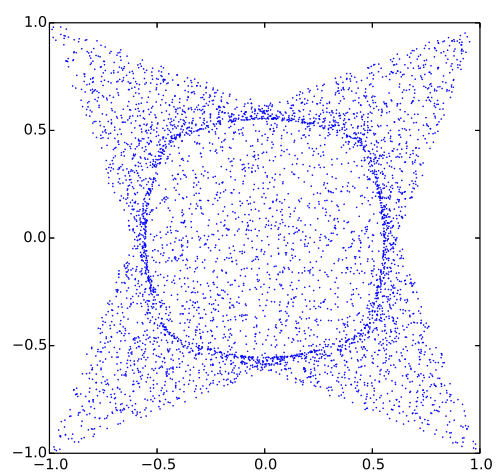

(b) $C_{P_{y}}\left(F_{n+2}\right)$ vs. $C_{P_{y}}\left(F_{n}\right)$

Figure 3: Chaotic correlations at Fibonacci times.

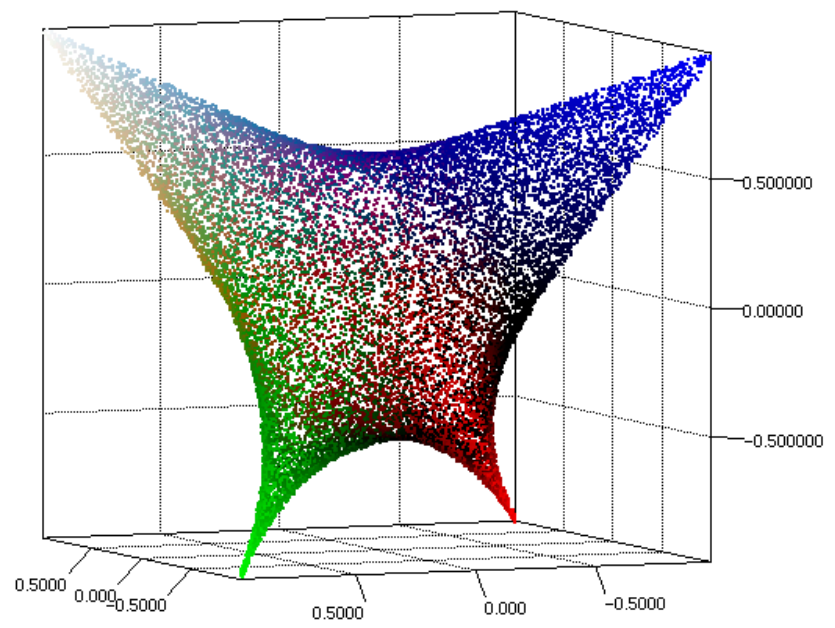

Figure 4: Plot of $C_{P_{y}}\left(F_{n+2}\right)$ vs. $C_{P_{y}}\left(F_{n+1}\right)$ vs. $C_{P_{y}}\left(F_{n}\right)$, revealing presence of invariant set embedded in three dimensional space.

The realization that the consideration of the functions $Z_{n}$ modulo $2 \pi / \kappa$ is sufficient to get a full understanding of the dynamics leads us to the following conclusions. First of all, to eliminate any rounding errors it is best to consider values of $\kappa$ which are a fraction of $\pi$, i.e. $\kappa=\pi / \gamma$ for some $\gamma \in \mathbb{N}$. From now onwards we will restrict ourselves to such choices. Secondly, for the class of modulation function under consideration, once the locations of the two discontinuities are known there are only a finite number of adaptations which can be made which could give different results. The first is the value of $Z_{1}(-X)$, and the second is the magnitude of the sizes of the discontinuities and both of these variables can take the values $0,1,2, \ldots, 2 \gamma-1$. When the two discontinuities have size zero this obviously leads to trivial behaviour of the ACF.

Let us put this idea into practice for $\kappa=\pi / 2$. Firstly, the value of $Z_{1}(-X)$ can only have three values leading to qualitatively different behaviour, as a choice of +1 or +3 both lead to the same value for $Q_{1}(-X)$ which is zero. 
Assume the magnitudes of the discontinuities are +1 or +3 and $Z_{1}(-X)=+1$ or +3 . We have $\cos (x \pm \pi / 2)=$ $\mp \sin (x)$ and $\cos (x \pm 3 \pi / 2)= \pm \sin (x)$, from which it follows that a region in which the function $Q_{n}(x)$ is positive or negative between two discontinuities is followed by a region where the function is zero. This can be seen as follows: If $Z_{n}(y)=+1$ or +3 , then $Q_{n}(y)=0$. Now we follow both functions until the next discontinuity, $y_{d}$ say, and so $Z_{n}\left(y_{d}+\epsilon\right)=Z_{n}(y) \pm(1$ or 3$)$ which is even. Hence $Q_{n}\left(y_{d}+\epsilon\right)=\cos \left(\frac{\pi}{2}\left(Z_{n}(y) \pm(1\right.\right.$ or 3$\left.\left.)\right)\right)=+1$ or -1 (depending on $\left.Z_{n}(y)\right)$. At the next discontinuity, $Z_{n}$ becomes odd again and the argument is repeated, sending $Q_{n}$ back to zero.

Using the modulation function

$$
\Phi(\phi)= \begin{cases}0, & x \in[0, \alpha] \cup[1-\alpha, 1], \\ +1, & \text { otherwise }\end{cases}
$$

we demonstrate what is described above. Figure 5 is a plot of $Q_{6}$ obtained when $\alpha=(\sqrt{2}-1) / 2$, showing the structure predicted. Iterating forward we numerically calculate the time series $\left(C_{P_{y}}\left(F_{n}\right)\right)$, and reconstruct the

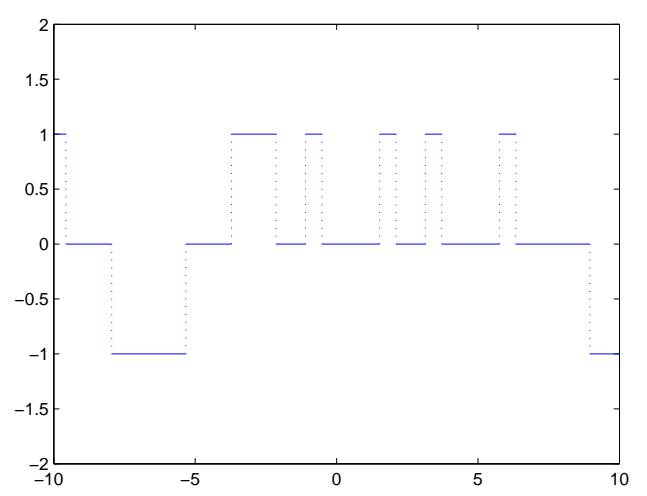

Figure 5: Plot of $Q_{6}$.

dynamics firstly in two dimensions and then in three dimensions. This is shown in Figures 6 and 7.

An interesting point to make is that if we assume the modulation has discontinuities of magnitude 1 or 3 , then regardless of $Z_{1}(-X)$ the correlations still appear to lie on the strange set depicted in Figure 7 . The only other choice for the magnitude of the discontinuity is 2 . Let us examine the modulation function given by $\hat{\Phi}=\Phi-1$ where $\Phi$ is as given by (4.3), with $\alpha \notin \mathbb{Q}(\omega)$ and $\kappa=\pi / 2$. Due to Table 1 we deduce that only every third Fibonacci number will give a value of the ACF other than zero for $\alpha / 2>\omega^{2}$ (for $\alpha / 2<\omega^{2}$ we have $Z_{1}(-X)=+3$ $(\bmod 4)$, but the same argument applies). The two- and three-dimensional projections are (as we would expect) simply crosses filling out the $x, y$, and in the latter case $z$, axes. It is intriguing instead to focus on the time series $\left(C_{P_{y}}\left(F_{3 n}\right)\right)$. The two- and three-dimensional projections are shown in Figure 8, showing again the presence of an invariant set embedded in three-dimensional space. 


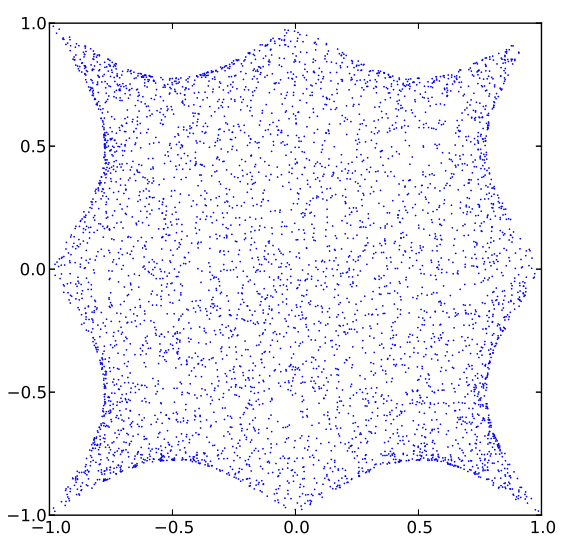

(a) $C_{P_{y}}\left(F_{n+1}\right)$ vs. $C_{P_{y}}\left(F_{n}\right)$

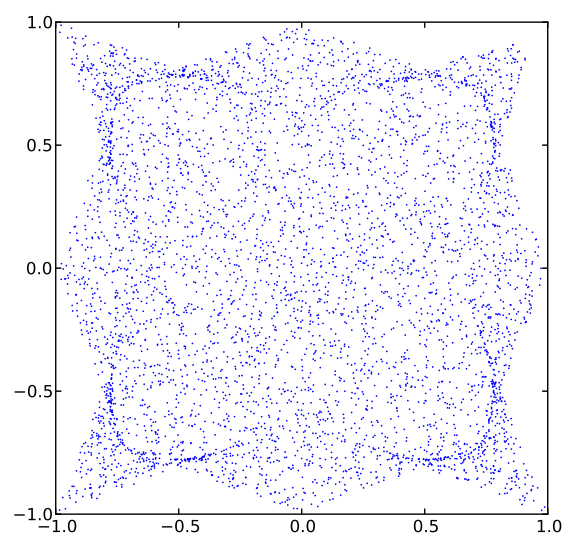

(b) $C_{P_{y}}\left(F_{n+2}\right)$ vs. $C_{P_{y}}\left(F_{n}\right)$

Figure 6: Two dimensional projections of the correlations at characteristic times plotted against one another.

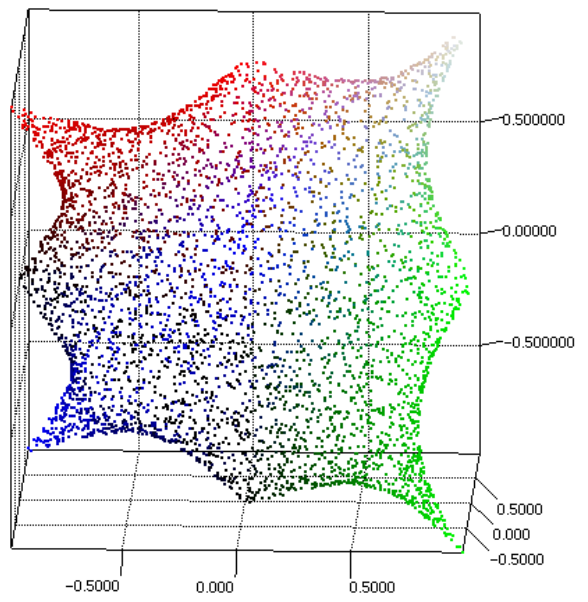

Figure 7: Plot of the invariant set in three dimensions with modulation function (5.1). 


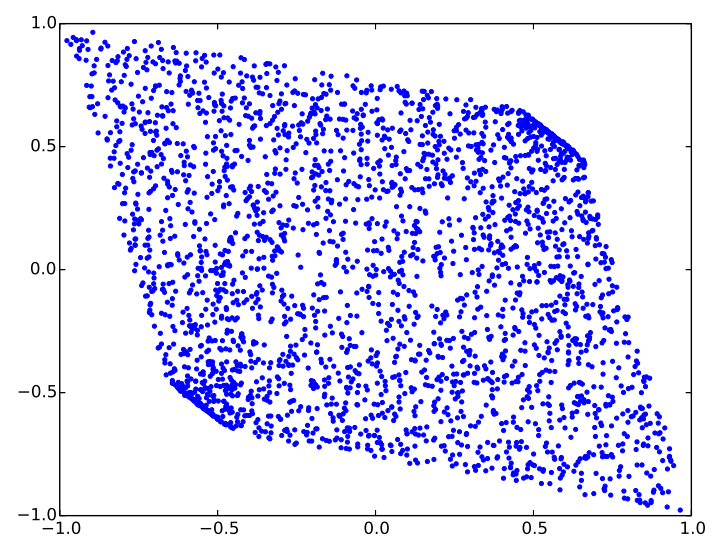

(a) $C_{P_{y}}\left(F_{3(n+1)}\right)$ vs. $C_{P_{y}}\left(F_{3 n}\right)$

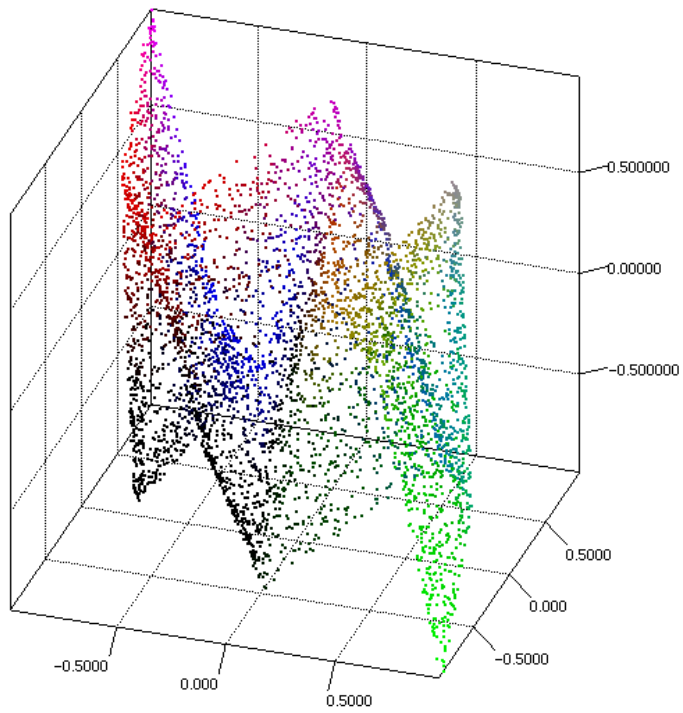

(b) $C_{P_{y}}\left(F_{3(n+3)}\right)$ vs. $C_{P_{y}}\left(F_{3(n+2)}\right)$ vs. $C_{P_{y}}\left(F_{3 n}\right)$

Figure 8: Numerical projections of the time series $\left(C_{P_{y}}\left(F_{3 k}\right)\right)$.

These projections can also be obtained in the barrier billiard system, but in this case the object is rotated due the signs of $Q_{n}(-X)$ and $\tilde{Q}_{n}(-X)$ not matching when $n \equiv 3(\bmod 6)$. Note that the same behaviour is seen if we select $Z_{1}(-X)=+3$.

\subsection{Varying the number of discontinuities}

To end this section we are going to give an example of some renormalization strange sets occurring when the modulation function has a greater number of discontinuities. We assume the discontinuities all have the same magnitude but with alternating sign, and as such the number of discontinuities must clearly be even. We take as an illustrative example $\kappa=\pi / 4$ and the modulation function given by

$$
\Phi(\phi)= \begin{cases}0, & 0 \leq \phi<\alpha / 4 \\ +2, & \alpha / 4 \leq \phi<\alpha / 2 \\ 0, & \alpha / 2 \leq \phi<1-\alpha / 2 \\ +2, & 1-\alpha / 2 \leq 1\end{cases}
$$

The resulting plots are shown in Figure 9 and we see once more the presence of renormalization strange sets. Other such sets can be found for other choices of $\kappa$ and for modulation functions with an even greater number of discontinuities, but this work is purely numerical and so in the next section we return to our assumption of two discontinuities.

\section{Varying the modulation amplitude/intensity $\kappa$}

We now focus on other values of $\kappa$. Let us begin with $\kappa=\pi / 3$, in which case we must work with $Z_{n}$ modulo 6 . Firstly, if we take $Z_{n}(-X)=0$ or $3(\bmod 6)$ and the modulus of the size of the discontinuities to be 3 (in the same 


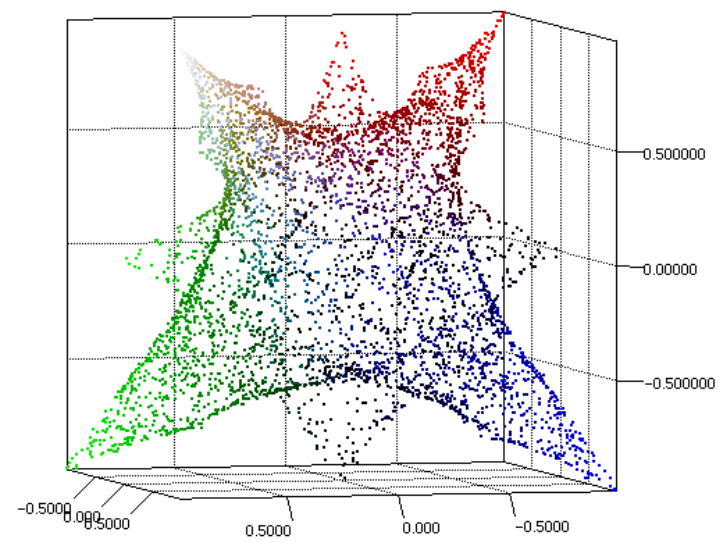

(a) $Z_{1}(-X)=0$

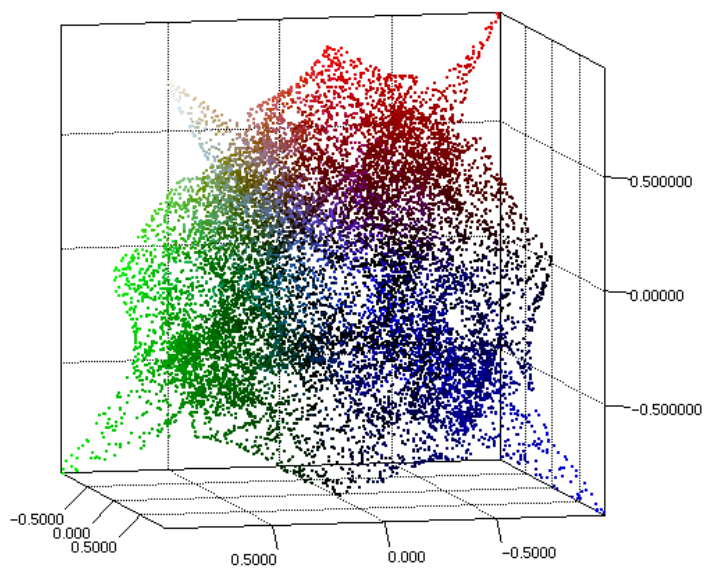

(b) $Z_{1}(-X)=2$

Figure 9: Renormalization strange set for modulation function with four discontinuities.

locations as (4.3), at $\alpha / 2$ and $1-\alpha / 2)$, then by the arguments of the previous section the correlations lie on the invariant set depicted in Figure 4. It should be clear how this generalises for $\kappa=\pi / \gamma$, in that we just simply pick $Z_{1}(-X)=\gamma$ or 0 , and the size of the discontinuities to be $\gamma$ and we are guaranteed to get the set in Figure 4 . Due to the fact that the function $\kappa \Phi(\phi)$ is what governs the dynamics (recall (2.8)), phenomena observed for certain choices of $\kappa$ can be observed for other values $\kappa$ but with the modulation function suitably scaled, and vice versa. For example, if we take $\kappa=\pi$ and $\hat{\Phi}(\phi)=\Phi(\phi) / 2$ where $\Phi$ is as in (4.3) then once again we get the renormalization strange set from Figure 4.

We now take $Z_{1}(-X)=1$ and note that $\cos (n \pi / 3)= \pm 1 / 2$ or \pm 1 depending on the value of $n$ modulo 6 . We pick once more the locations of the discontinuities to be the same as that in the study of the $\alpha$-barrier (at $\alpha / 2$ and $1-\alpha / 2)$ to get a clearer understanding of the resulting phenomena. We assume the discontinuities have magnitude 3. Clearly, every time we hit a discontinuity of $Z_{n}(x)$ it has the effect of multiplying $Q_{n}(x)$ by -1 . So we have piecewise constant functions taking the values \pm 1 only or $\pm 1 / 2$ only, depending solely on $Z_{n}(-X)(\bmod 6)$. As the discontinuities are in the same positions as for the barrier billiard system, this means that for each value of the ACF is either the same or half of the value obtained for barrier billiards (up until a sign choice). If we iterate (4.2) modulo six with $Z_{1}(-X)=1$ we find that a period 24 orbit results of $Z_{n}(-X)$, and which gives $Q_{n}(-X)$ the pattern $\pm 1, \pm 1 / 2, \pm 1 / 2, \pm 1 / 2, \pm 1, \ldots$. As we get a sequence where there are no consecutive \pm 1 , we can conclude that the projections of Figure 3 will not be present, but there will instead be three scaled and stretched copies.

The first copy will be the original but scaled by factor $1 / 2$ as we have the presence of two consecutive $\pm 1 / 2$ 's. If we have a $\pm 1 / 2$ followed by a \pm 1 then we have one point lying on the first copy and the second on the original creating a vertical stretch by a factor of 2 of the first copy, giving the second copy. Finally, for \pm 1 followed by $\pm 1 / 2$ we clearly have a horizontal stretching of the first copy giving the third copy. A similar argument of the set of possible sign triples of $Q_{n}(-X)$ tells us that (allowing for symmetry) the three dimensional projection will just be (at least) four scaled versions of the original in Figure 4. These two and three dimensional projections are shown in Figures 10 and 11. 


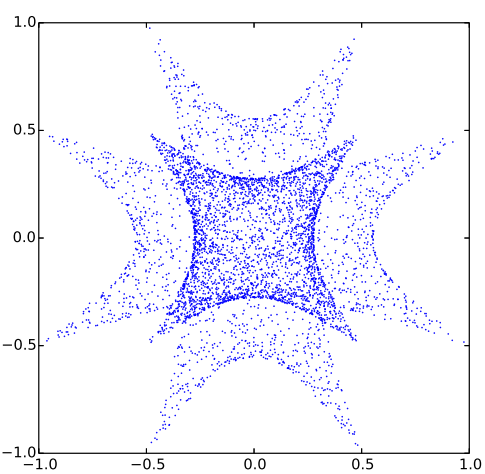

(a) $C_{P_{y}}\left(F_{n+1}\right)$ vs. $C_{P_{y}}\left(F_{n}\right)$

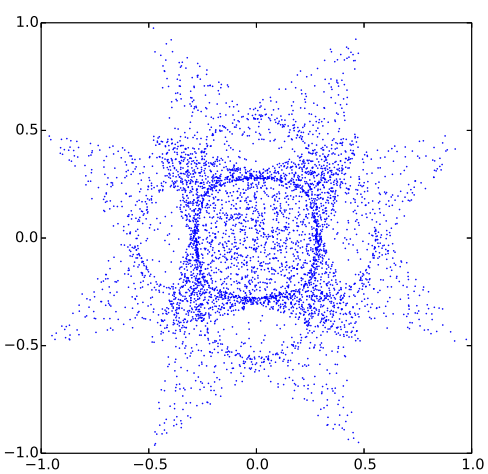

(b) $C_{P_{y}}\left(F_{n+2}\right)$ vs. $C_{P_{y}}\left(F_{n}\right)$

Figure 10: Two dimensional projections of the correlations at characteristic times plotted against one another.

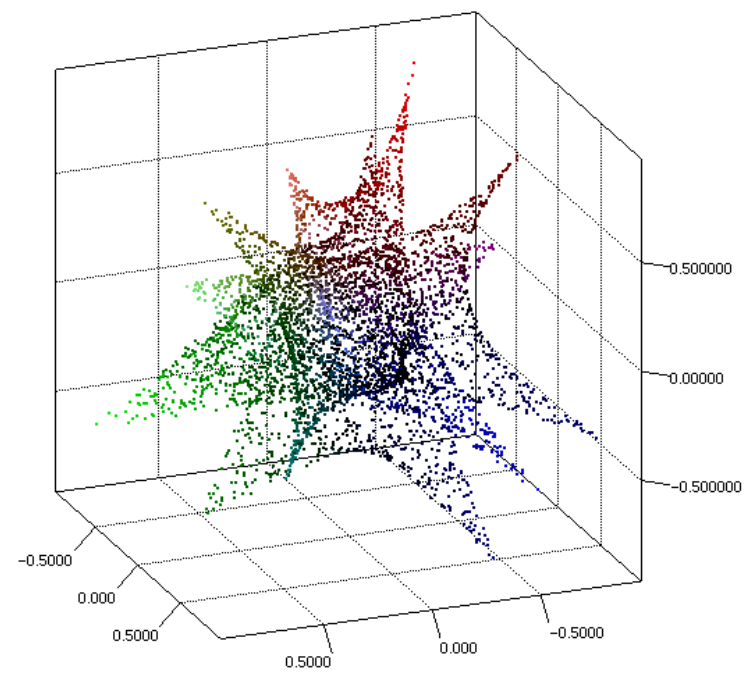

Figure 11: Plot of the invariant set in three dimensions with modulation function (5.1).

This same picture is observed for $Z_{1}(-X)=2,4,5$ and this thus exhausts the options when the discontinuity size is 3 .

If $Z_{1}(-X)=+1$ and the discontinuities have magnitude 2, we get the projections shown in Figure 12. We see concentrated lines of points caused by the autocorrelation function repeatedly returning to $\pm 1 / 2$, forming a square. The reason for this is the presence upon iteration of (3.7) of functions $Z_{n}$ (modulo 6) taking the successive values $1,5,1,5, \ldots$ and $2,4,2,4, \ldots$, which makes $Q_{n}(x)$ a constant function taking the value $\pm 1 / 2$. Two examples of such functions are shown in Figure 13. 


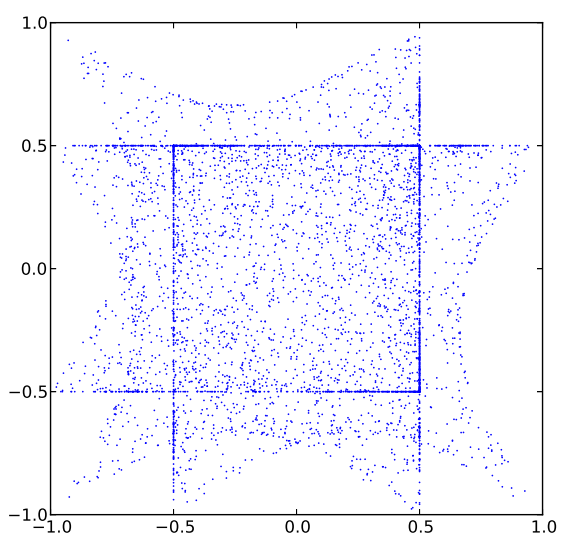

(a) $C_{P_{y}}\left(F_{n+1}\right)$ vs. $C_{P_{y}}\left(F_{n}\right)$

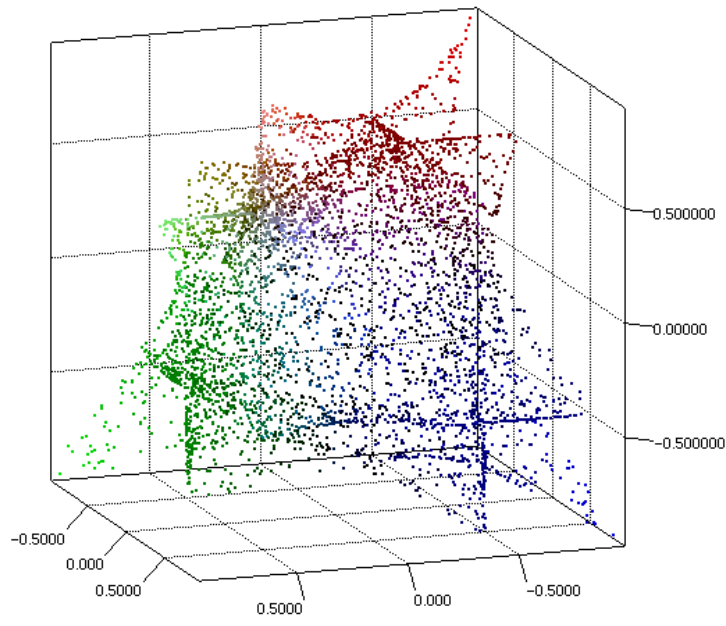

(b) $C_{P_{y}}\left(F_{n+2}\right)$ vs. $C_{P_{y}}\left(F_{n+1}\right)$ vs. $C_{P_{y}}\left(F_{n}\right)$

Figure 12: Two and three dimensional projections of the correlations at characteristic times plotted against one another.

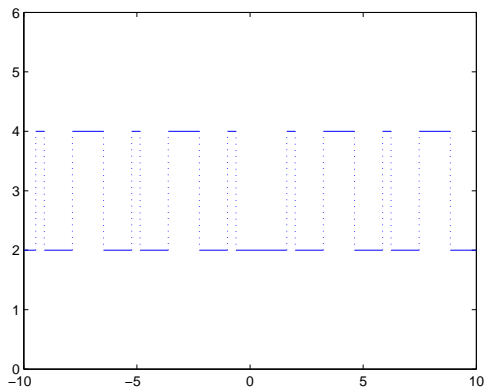

(a) Plot of $Z_{17}(\bmod 6)$

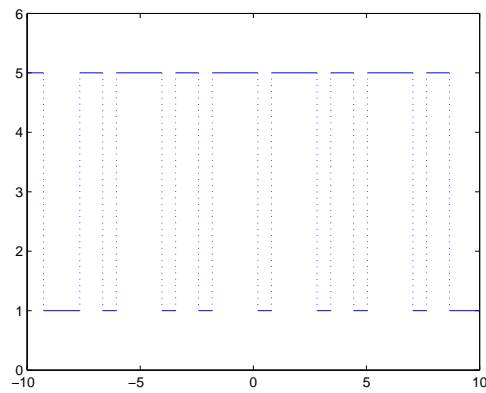

(b) Plot of $Z_{30}(\bmod 6)$

Figure 13: Behaviour of $Z_{n}$ which causes the $\mathrm{ACF}$ to take the value $\pm 1 / 2$, using initial modulation function $\tilde{\Phi}(\phi)=\Phi(\phi)-1$ where $\Phi(\phi)$ is defined in equation (4.3).

If we take $\kappa=\pi / 4$ and leave everything else the same, then we can expect a similar phenomena. In this case we take $Z_{n}(\bmod 8)$ and find that obtain functions similar to those shown in Figure 13 (not the same iterate numbers generally) but which take the values 1 and 7 only or functions taking the values 3 and 5 only, which make the autocorrelation function equal to $\sqrt{2} / 2$, and so we get the two dimensional projection shown in Figure 14 again with a square of points where the ACF takes this value.

\subsection{Small relative magnitude discontinuity sizes}

In this subsection we are going to explore what happens if the size of the discontinuities is small in comparison with $\gamma$ for the class of modulation function under study. With these assumptions we observe convergence to the 


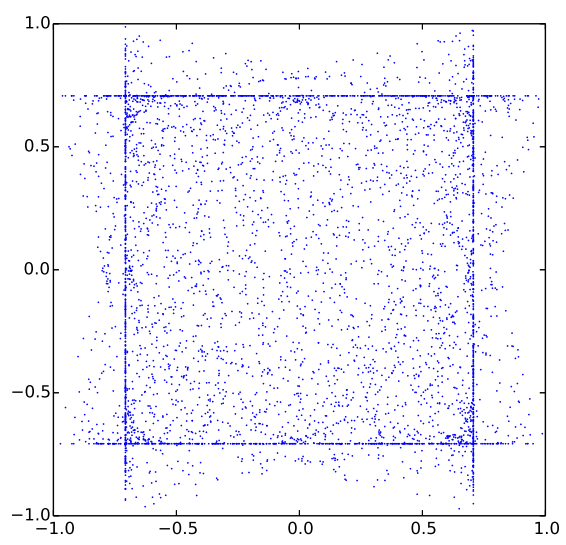

Figure 14: Two dimensional projection obtained for $\kappa=\pi / 4$ with $Z_{1}(-X)=1$ and discontinuity magnitude 2

renormalization strange set shown in Figure 15.

Of course as $\gamma \rightarrow \infty$ we can pick the discontinuity sizes to be a large as we desire, as long as they are small with respect to $\gamma$. So in some ways this can be looked at as the set occurring in the limit $\kappa \rightarrow 0$, although it can be observed for any value of $\kappa$ with small enough discontinuity sizes. Note that this only occurs in the limit $\kappa \rightarrow 0$, because at $\kappa=0$ the ACF is trivially +1 , so the limit is singular.

\section{Conclusion}

We have extended the results of previous work on this system to a general class of modulation function, in particular the class of modulation function $\Phi$ with only two discontinuities. It is demonstrated that for a modulation function whose discontinuities lie in $\mathbb{Q}(\omega)$ we can expect periodic behaviour of the ACF, or if this condition is not satisfied, chaotic behaviour of correlations on an invariant set embedded in three dimensional space.

The key to this numerical work has been the elimination of the issue of unbounded orbits of the renormalization operator through the consideration of such orbits modulo an integer which is related to the intensity of modulation $\kappa$ when $\kappa$ is selected to be a rational multiple of $\pi$. This enables numerical approximation of the ACF and thus the construction of the renormalization strange sets. It is shown that once the locations of the discontinuities of the modulation function have been established, only a finite number of adaptations can be made which yield topologically different strange sets. These alterations are the value of the modulation function at an endpoint and the size of the two discontinuities, which themselves have only a finite number of variants. It is also demonstrated that the correlations continue to lie on such strange sets for modulation functions with more than two discontinuities.

The work also provides a link between the two-level system and other quasi-periodically forced systems. We have shown that the renormalization strange set occurring for symmetric barrier billiards can be reproduced in the two level system, and in [1] we found the same set occurring in the study of correlations of strange non-chaotic attractors, thus making it a somewhat universal set in the study of correlations for this class of system. This work can be trivially generalised to a class of quadratic irrational frequency as has been done in the past [15], although the problem of generalising to general irrational frequencies remains challenging.

In future works we hope to be able to produce an analytic proof of the chaoticity of the correlations for symmetric barrier billiards (which lie on the set shown in Figure 4) by conjugating the renormalization operator to a sub-shift of finite type. If successful, this will naturally carry over to the work presented here for the two-level system and 


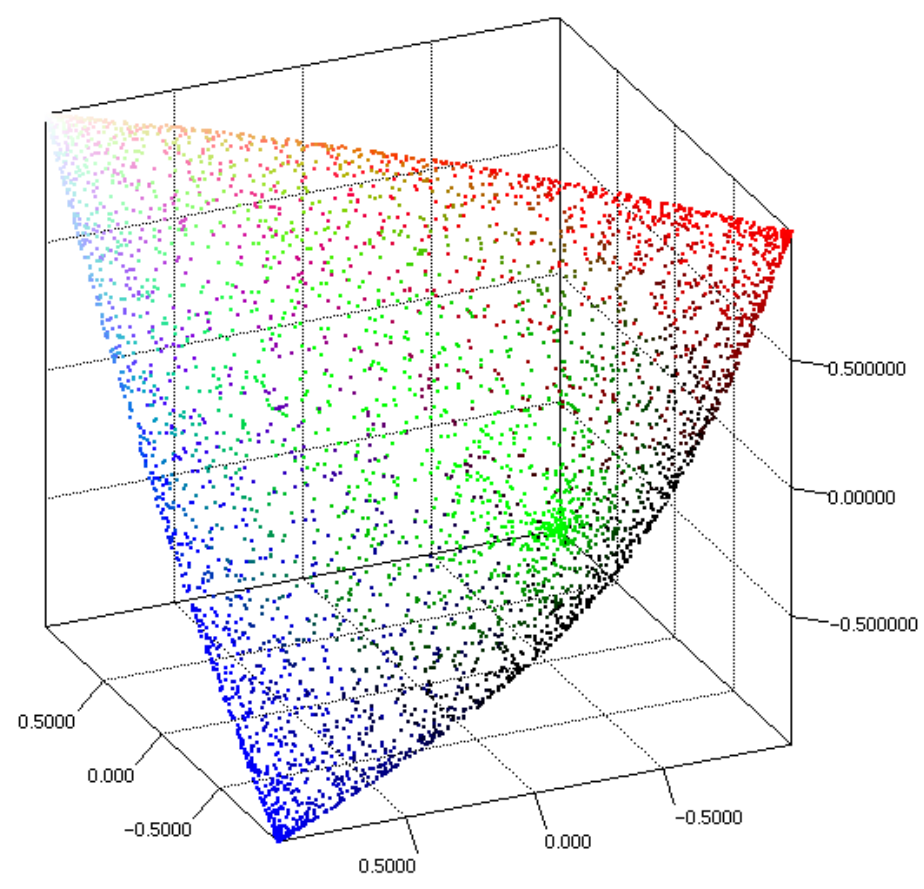

Figure 15: Renormalization strange set which appears in the limit $\kappa \rightarrow 0$.

the work presented in [1] for the correlations of SNAs.

\section{References}

[1] L N C Adamson and A H Osbaldestin, The non-smooth pitchfork bifurcation: A renormalization analysis, Submitted to Dynamical Systems Journal.

[2] _ Renormalisation of correlations in a barrier billiard: Quadratic irrational trajectories, Physica D: Nonlinear Phenomena 270 (2014), 30-45.

[3] J R Chapman and A H Osbaldestin, Self-similar correlations in a barrier billiard, Physica D 180 (2003), no. 1, $71-91$.

[4] J Dalton and B D Mestel, Renormalization for the Harper equation for quadratic irrationals, Journal of Mathematical Physics A 44 (2003), 4776-4783.

[5] U Feudel, A Pikovsky, and A Politi, Renormalization of correlations and spectra of a strange non-chaotic attractor, Journal of Physics A: Mathematical and General 29 (1996), no. 17, 5297-5311.

[6] U Feudel, A S Pikovsky, and M A Zaks, Correlation properties of a quasiperiodically forced two-level system, Physical Review E 51 (1995), no. 3, 1762-1769.

[7] C. Grebogi, E. Ott, S. Pelikan, and J. A Yorke, Strange attractors that are not chaotic, Physica D 13 (1984), no. $1,261-268$.

[8] J A Ketoja and I I Satija, Self-similarity and localization, Physical Review Letters 75 (1995), no. 14, 2762-2765.

[9] S P Kuznetsov, A S Pikovsky, and U Feudel, Birth of a strange nonchaotic attractor: A renormalization group analysis, Physical Review E 51 (1995), no. 3, R1629-R1632. 
[10] J M Luck, H Orland, and U Smilansky, On the response of a two-level quantum system to a class of timedependent quasiperiodic perturbations, Journal of statistical physics 53 (1988), no. 3-4, 551-564.

[11] B D Mestel and A H Osbaldestin, Periodic orbits of renormalisation for the correlations of strange nonchaotic attractors., Mathematical Physics Electronic Journal [electronic only] 6 (2000), no. 5, 27.

[12] _ Renormalization analysis of correlation properties in a quasiperiodically forced two-level system, Journal of Mathematical Physics 43 (2002), 3458-3483.

[13] __ A garden of orchids: a generalized harper equation at quadratic irrational frequencies, Journal of Physics A: Mathematical and General 37 (2004), no. 39, 9071-9086.

[14]__ Golden mean renormalization for a generalized Harper equation: The Ketoja-Satija orchid, Journal of Mathematical Physics 45 (2004), 5042-5075.

[15] __ Renormalization of correlations in a quasiperiodically forced two-level system: quadratic irrationals, Journal of Physics A: Mathematical and General 37 (2004), no. 38, 8969-8989.

[16] B D Mestel, A H Osbaldestin, and B Winn, Golden mean renormalization for the Harper equation: the strong coupling fixed point, Journal of Mathematical Physics 41 (2000), 8304-8330.

[17] A H Osbaldestin and L N C Adamson, Chaotic correlations in barrier billiards with arbitrary barriers, Journal of Physics A: Mathematical and Theoretical 46 (2013), no. 24, 245101.

[18] A S Pikovsky and U Feudel, Correlations and spectra of strange nonchaotic attractors, Journal of Physics A: Mathematical and General 27 (1994), no. 15, 5209-5219.

[19] M T Rosenstein, J J Collins, and C J De Luca, A practical method for calculating largest Lyapunov exponents from small data sets, Physica D: Nonlinear Phenomena 65 (1993), no. 1, 117-134.

[20] F Takens, Detecting strange attractors in turbulence (Lecture Notes Mathematics vol 898), Berlin: Springer (1981), 366-381.

[21] J Wiersig, Singular continuous spectra in a pseudointegrable billiard, Physical Review E 62 (2000), no. 1, $\mathrm{R} 21-\mathrm{R} 24$. 